\title{
Enhanced Content Detection Method to Detect Online Spread of Terrorism
}

\author{
J. Kiruba, P. Sumitha, K. Monisha, S. Vaishnavi
}

\begin{abstract}
Terrorist growth reached to certain part of the world. Terrorist groups have spread their information on social network like facebook, whatsapp, messages. Before spreading the terrorism activities, we need to change and abort it. The basic idea is to stop or reduce spreading of terrorism is to remove all this accounts. Web is a noteworthy wellspring of spreading psychological warfare by discourse, content, sound arrangement. Terrorists use internet to persuade individuals and it paves the way to involve in terrorist activities through provocative webpage that inspires individuals to involve in terrorist groups and organization. To execute this thought we need a great deal of human exertion to gather the data and discover the fear monger gatherings. To reduce the human effort, we implement the system which detects terrorist groups in social media. To implement this idea we are in need of a lot of human effort to gather the information and find out the terrorist groups who are involved in. Thus we implement this system for major causes which detects terrorist groups in internet and social media. It paves the way to reduce the human effort.
\end{abstract}

\section{INTRODUCTION}

Terrorism is widely spread and grown deeply in some areas all around the world. It is very important to control terrorism and to stop it before spreading before some certain period of time. The major source of spreading out terrorism is internet through media such as images,speeches,videos and url's. Terrorism Groups use web to persuade people and youth and it also influence individuals to involve in terrorist activities through alluring web pages which inspire people and youth to involve in terrorist organization. Thus, the proposed and efficient techniques by using segmentation technique. It flags those activities automatically for human review. It is difficult to study for single algorithm in websites because the websites created in various platforms use different data structures. so we use DOM Tree idea to separate the web information and SIFT highlight for edge extraction that sorted out web information. Also we use KMeans algorithm for segmentation and KNN for classification. We use KNN algorithm for classification and $\mathrm{K}$-means algorithm for Segmentation. There is a need to find that web pages promoting terrorism or not promoting. This system is useful in anti-terrorism activities and even in search engines to divide the websites. The result provided by to find terrorist suspected in terrorism activities.

Revised Manuscript Received on 14 August, 2019.

J. Kiruba, Assistant Professor, Karpagam College of Engineering, Coimbatore, Tamilnadu, India. (Email: kirubajesudasan@gmail.com)

P.Sumitha,Student, Karpagam College of Engineering, Coimbatore, Tamilnadu, India. (Email: sumithaprabhakaran14@gmail.com)

K.Monisha,Student, Karpagam College of Engineering, Coimbatore, Tamilnadu, India. (Email: rkmoni97@gmail.com)

S.Vaishnavi, Student, Karpagam College of Engineering, Coimbatore, Tamilnadu, India. (Email: vaishukumar375@gmail.com).
ISP's traffic methodology is used to detect users related information who involves in terrorist. The criteria for the proposed systems are:

1.The algorithm for detection environment should be implemented based on the information of Terrorist sites in existing period in web.

2.The process of detection should be done in real time. This can be implemented only when information presented in a compact manner for existing processing.

3.According to the user-defined parameters the detection sensitivity is managed by provoke calibration of the performance of desired direction

\section{PROBLEM STATEMENT}

In this paper, the real time nature of website is investigated which is designed to check whether a valid information can be extracted from it. They proposed system deliver event notification which is used to monitor the activities and delivers notification according to the investigation knowledge. This research have three stepsFirst, According to the target event, they have numerous activities based on crawl. Second, they estimate location of events and propose probabilistic activities to extract events. Finally, Alert reporting system is developed that takes earthquakes from websites and message is sent the registered user.

\section{METHODOLOGY}

Here ,they explained the methods as a target event using an earthquake. It is restricted for websites. Prominently classification is the undertaking of making a qualification between genuine client and a psychological militant is a noteworthy arrangement issue with two alternative classes as terroristsvs non-terrorists. Although, the most common classification algorithm called as Native Bayes are naturally probabilistic. That is they accepted and likelihood dispersion of classes in a stable and moderately adjusted classes over population. Moreover, they as a rule overlook the unmistakable between the misclassification article costs having a place with various classes. All those assumption are totally wrong while they involving with terrorist detection on the internet. The monitored population is unbalanced so that entire population of internet users is actually close to low, that is zero. It is not expected that the terrorists activities on the internet to be a stable either-in

Published By: 
fact .In web Activities, it is interested in any fluctuations in those web activities and it missing one real terrorists in haystack of user may be highly affected than suspecting several users of being active participant of terrorists. Apparently, it is decided to follow the clustering approach which is more flexible to detect terrorism, while involving the investigation of classification methods for further research

\section{CLUSTER METHOD}

It is a method of partitioning meaningful group of clusters from data objects. After the clusters have a same similarity based on characteristics or vice-versa. Clustering is an unsupervised division of patterns(data items)which is unlabelled and there is no pre-defined labels Which is associated with set of objects .This results in compact of high sets by tiny cluster centroids. Clustering application includes text document retrieved, classification of patterns, mining such as text and data and segmentation of image .Some of the clustering detection techniques :By using similar cluster, we can find normality of an objects. Clustering, is done by retrieving web pages from terroristrelated sites and is used to find starts of web pages consist to the same terrorists. A centroid found and showed by the vector model for each and every stack. Whenever there is a similarity between closest centroid and have higher threshold using access vector, an alarm is issued by the detection.

\section{DARK WEB PREDICTION}

The manual task for prediction of dark web is less efficient and will take more time due to huge data availability .So, there is need of an automated system that performs the prediction of dark web. Fig 1 shows the terrorist attack between years between 2000-10 that happened all over world. We show that terror related activities are in high number over the time by analyzing this graph. This indicates that it is the terrorism is the biggest problem for a country and making of recruitments of new member and there are various dark websites used by terrorist.

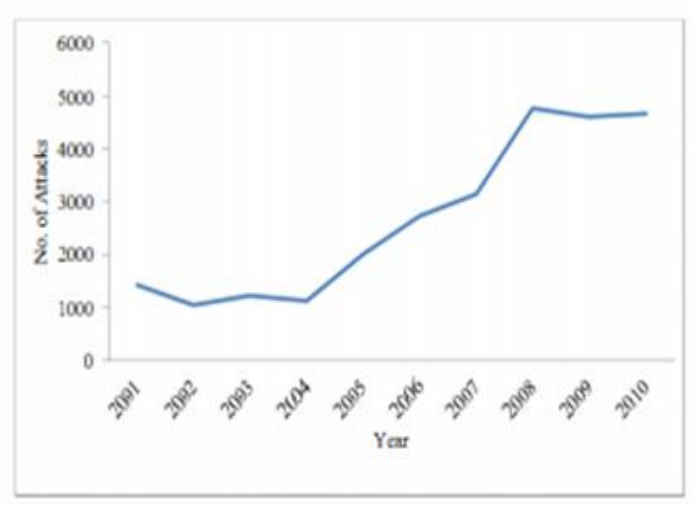

Figure 1. Terrorist Attacks

\section{EXISTING SYSTEM}

The Existing system, there was no such system to keep an eye on various websites or any suspicious words present online. Cops were unable to track the terrorist related website or any person with suspicious information. The proportion of fear mongering is high in this day and age. There must be a framework to follow those suspicious word on the web and cut down the proportion of fear based oppression.

\section{DISADVANTAGES OF TERRORISM SITE ACTIVITIES}

The disadvantages of existing system is if terrorist used their eavesdropping in codewords. By codewords, we can't able to find the terror-related websites

The detecting system is use to constantly monitor actions in certain environment. This system decides whether they are part of a legitimate use of the environment or a possible hostile Attack. The error from the environment in that several computer attached to a network other same network. The terror-related contents are view more specifically. The prediction of similarity is currently against the accessed page.

\section{PROPOSED SYSTEM}

In proposed system, to mine textual information on webpage and find their similarity to terrorism, we designed web mining techniques. By detecting websites and check them of theymany be promoting terrorism. This paper shows useful in search engines to classify websites into the category and anti-terrorism. The division is based on the prediction to the fields and arrange them approximately and result in human reviews. By real-time scenario, the detection is takes places. It is achieved by efficient system by Knowledge of codeword used by terrorists.

The criteria for this proposed system are:

1. This algorithm based on existing terror-related activities sites and contents from the existing system and known traffic webs.

2. Based on real time, the detection is result out. If the result of information interacts gives the achievement of goals and process in a efficient manner.

3. To empower calibration for the presentation of recognition affectability ought to be constrained by client characterized data.

\section{DETECTION BASED ON CONTENT- DETECTING ENVIRONMENT}

By using content of various websites, we suggested a new detection techniques. The inputs are taken from terrorists and supporters to process their terror-related activities. This paper suggest contextual reports and other complex data's. The terrorist activities and their supported activities are viewed and monitored by using learning process to predict typical views of terrorists. It is used for future detection of terror-related activities caused by terrorists and groups of supporter cells.[5]The main concept of our system is to monitor terrorist by their system IP's and passed message by certain authorities. And the easy detection of terrorist can suspect by maximum time of log in with their own system. 


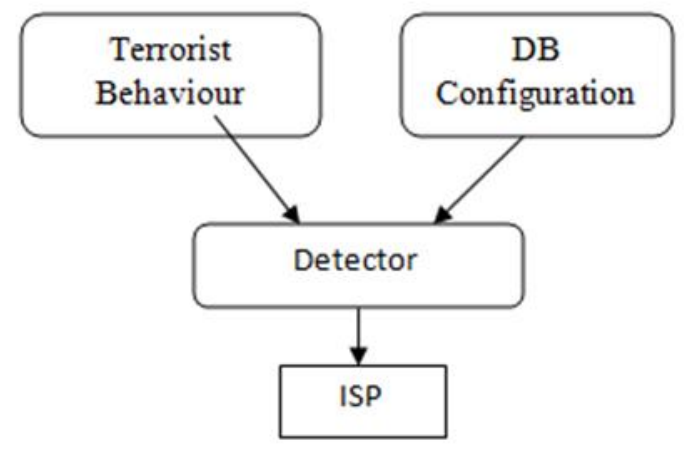

Fig 1:DETECTING ENVIRONMENT

\section{LEARNING THE BEHAVIOUR OF TERRORIST}

The terrorist behaviour is indentified by web services by their secret patterns and Keywords. Figure 2 describes the module for learning. The assumptions of terror related activities collected by websites ,the collector information can be generated by vector data method. The converted Vector data has been changed to access and centroid vector terms .The data is stored in Transactional databases for future use. The clustering method is used to detect the unclustered data into clustering format of data. This clustering method finds terrorist activities and computes centroid vector techniques. The behaviour of terrorist is resulted by a group of centroid vector data items which represents the set of terrorist interests.

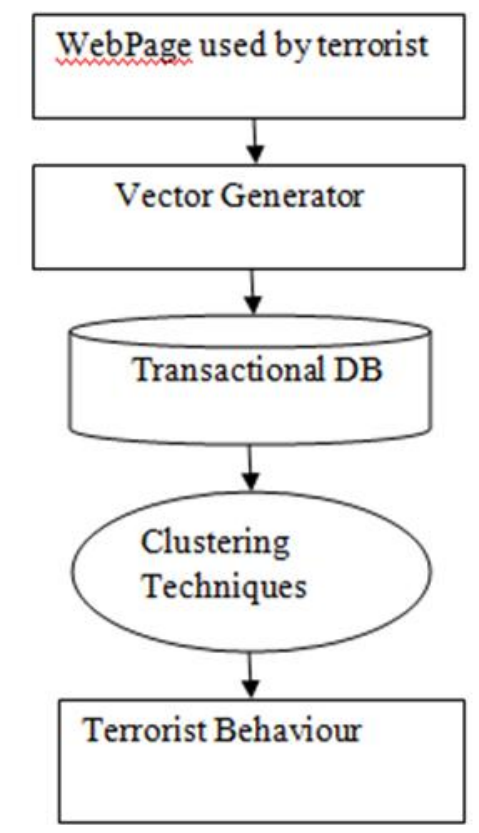

\section{Fig.2 LEARNING TERRORIST BEHAVIOUR}

\section{TERRORIST DETECTING METHOD}

This terrorist detecting method is used to monitor the private conversation of terrorist by generating access vector method by IPS Traffic signal. The detector which is used to detect the vector and need to find that vector is found by terrorist. There is a two vector as Access an centroid which find similarity between two methods. For finding similarity, we used cosine formula.

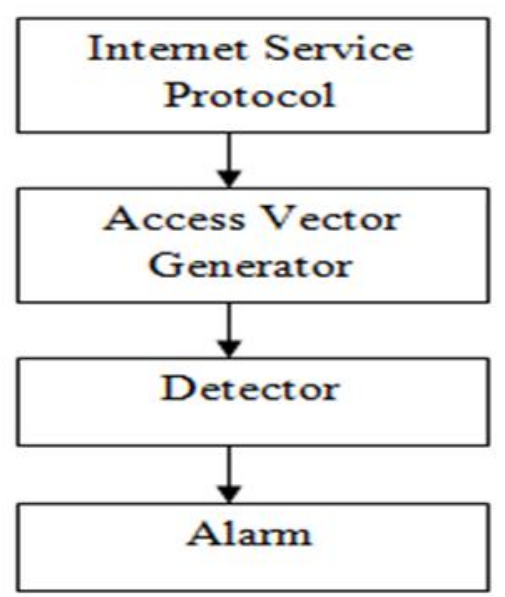

TERRORIST DETECTING METHOD

\section{RELATED WORK}

In earlier system, the terrorist used the Infrastructure by Internet to exchange their private conversation as terrorrelated activities and schedule it by using more new groups and supporters[2]. The techniques used to detect the terrorist by eavesdrop by using Internet protocol which is using by terrorists. But, it is difficult to manage and monitor the terrorist IP address and url which is used to filter keywords and patterns. And there is a frequency change of Location of web server and there is no fixed IP Address. To overcome this disadvantages, there is a law which using ISP traffic through privacy issues. The integrated issues which is done by earlier using fields of Computer Security and retrieval of Information and mining by texting mechanism[4].

\section{RESULT}

This paper presents the detection of web based terrorist activities methods and Knowledge. This study results the concepts and methods to detect terrorists and their behaviour and their supporters who supports for terror related activities in society using an Internet access.

\section{DISCUSSION}

Psychological oppressor associations are utilizing the web to spread their purposeful publicity and radicalize youth on the web and urge them to submit fear based oppressor exercises. To reduce the online foot print of such harmful websites we need to create a system which detects specific keywords in that particular website and if those keywords are found then that website should be blacklisted The development for efficient system uses two mining methods for detect terror-related activities as called data and web based mining . Text Mining is one of the concepts of Web Mining techniques. Its function is to extract and scan the unstructured data contents. The another efficient techniques is to find patterns and keyword in unstructured data contents. 
[2] The terrorists can collects the information for Infrastructure of Internet sources and spread their activities by recruiting new groups and supporting hands.The way to find the terror-related which is done by terrorist using IP address of their systems. And the other ways to hack their private conversations with terrorist organization by social medias. But their won't use the fixed Internet Protocols for sharing their information privately. And Also their is a frequency changes of locations in web services for their eavesdropping.

[3] There is an application which is use to detect IP address used by terrorist for terror-related activities using Social Network. This system helps to detect terrorist by filtering mechanism to overwhelmed useless websites. This filtering mechanism is used to filter out the unwanted information and there is need to auto-control the message.

\section{CONCLUSION}

To check the threat of psychological oppression and to pulverize the online nearness of hazardous fear mongers associations like ISIS and other radicalization sites. We need a legitimate framework to detect and end sites which are spreading hurtful substance used to radicalizing youth and powerless individuals. This Project have been implement Web based Platform and Also, different attributes have been added to the project which will prove to be advantageous on the real time application of the system. This application can be implemented by any admin manager for distributing it to their administrators who can detect the spread of terrorism.

This paper presents the detection of web based terrorist activities methods and Knowledge. This study results the concepts and methods to detect terrorists and their behaviour and their supporters who supports for terror related activities in society using an Internet access.

\section{REFERENCES}

1. Dietmar Jannach and Simon Fischer, "Recommendationbased Modeling Support for Data Mining Processes", Germany and Simon Fischer Rapud Miner GMBH Germany, Proceedings of the 8th ACM Conference on Recommender System, October-2014.

2. Jiawei Han Micheline Kamber Jian Pei,'Data Mining Concepts and Techniques" 3rdEdition,June 2011.

3. T.Sumil Kumar, Dr.K.Suvarchala, "A Study of Web Data Mining Challenges and Applications for Information Extraction",November-2012.

4. Syed Ahsan, AbadShah, "Data Mining ,Semantic Web and Advanced information Technologies for Fighting Terrorism",June-2013.

5. http://www.ise.bgu.ac.il/faculty/mlast/papers/jiw_paper.p df

6. http://citeseerx.ist.psu.edu

7. A Student of RTMNU University, "Web Data Segmentation for Terrorism Detection using Named Entity Recognition Techniques", June 2017.

8. Mr.AnilKumarMunani,Mr.BhaveshTanawala,Mr.Prashan tSwadas,"A Survey Prediction on Online Spread of Terrorism",February-2018.

9. V.Elovivi,A.Kandel,M,Last,"Using Data Mining Techniques for detection Terror-Related Activities on the Web,April-2015.

10. Spafford,E.H.Zamboni,"Intrusion Detection using autonomous agents", March 2003. 